\title{
Research Based on Lee Algorithm and Genetic Algorithm of the Automatic External Pipe Routing of the Aircraft Engine
}

\author{
Qian Zhou", Yanjie $\mathbf{L v}^{2, *}$ \\ ${ }^{1}$ National Laboratory for Aeronautics and Astronautics, Beihang University, Beijing, China \\ ${ }^{2}$ Aerospace Information Research Institute, Chinese Academy of Sciences, Beijing, China
}

Email address:

zhouqian@buaa.edu.cn (Qian Zhou), lvyanjie@126.com (Yanjie Lv)

${ }^{*}$ Corresponding author

\section{To cite this article:}

Qian Zhou, Yanjie Lv. Research Based on Lee Algorithm and Genetic Algorithm of the Automatic External Pipe Routing of the Aircraft Engine. International Journal of Mechanical Engineering and Applications. Vol. 8, No. 1, 2020, pp. 40-44. doi: 10.11648/j.ijmea.20200801.16

Received: January 16, 2020; Accepted: February 7, 2020; Published: February 20, 2020

\begin{abstract}
The casing of aeroengine has the characteristics of rotating surface, and more than one hundred pipes are decorated between the casing and outer surface of the nacelle. For the narrow space and various constraints of pipe routing, manual pipe routing is not only labor intensive, but also difficult to ensure the accuracy. To resolve the problem of automatic pipe routing of the aircraft engine, a novel method based on lee algorithm and genetic algorithm is proposed. Firstly, a 3D environment of pipe routing is built and then the initial population for the genetic algorithm is formed by lee algorithm combined with roulette method. Each chromosome of the population represents a possible solution of pipe routing. Secondly, a variable length coding scheme based on chain table is used for chromosome encoding. The genetic manipulation includes selection, crossover and mutation. This paper presents the fitness function based on the constraint condition of pipeline routing. Finally, the useful and feasibility of this method is developed and verified by developing an automatic pipe routing module based on UG and VC.
\end{abstract}

Keywords: Aircraft Engine, Pipe Routing, Lee Algorithm, Genetic Algorithm

\section{Introduction}

Pipe routing is important in aeroengine design. The external pipes of aeroengine are complex and numerous, including fuel pipes, lubricating oil pipes, air pipes, control hydraulic pipes, cables and so on [1]. A good pipe system is important for the reliable and safe of the aeroengine and effects the cost and performance. For the narrow space and various constraints of pipe routing, manual pipe routing is not only labor intensive, but also difficult to ensure the accuracy [2-3].

Since the 1970s, many scholars at home and abroad have begun to apply computer-aided technology in pipe routing and study the problems about automatic pipe routing method and gained some achievements [4-7]. The main methods used in pipe routing such as escape algorithm, network optimization algorithm, cell generation algorithm, ant colony algorithm and so on [8-19]. For the complexity of pipe routing environment, mature theories and methods have not been formed in pipe laying yet. Escape algorithm can not find the shortest path sometimes. Network optimization algorithm needs to choose a series of intermediate nodes before generating pipeline. Genetic algorithm usually generates illegal result which leads a lot of repair work. Cell generation algorithm considers too many constraints in its evaluation function to establish the basic path when the pipe laying space is complex. Ant colony algorithm is difficult to initialize the digital model for pipe routing [20-22].

This paper combines genetic algorithm and lee algorithm, and adopts the variable length coding scheme based on chain table for automatic pipe routing. To verify the feasibility of this method, an automatic pipe routing module is developed based on the platform of UG.

\section{Problem Description}

The casing of aeroengine has the characteristics of rotating surface, and more than one hundred pipes are decorated between the casing and outer surface of the nacelle. The 
space for pipe routing is narrow. According to the characteristics of the rotating surface of the engine case, a series of cylinders can be used to approximate the rotating surface, and the space between the two cylinders can be used to represent the pipe routing area. The surface of the casing of aeroengine is defined as $\mathrm{C} 1$ and the radius is $\mathrm{R} 1$. The outline surface of aircraft engine nacelle is defined as $\mathrm{C} 2$ and the radius is $\mathrm{R} 2$. The axis of $\mathrm{C} 1$ is defined as $\mathrm{Z}$ axis. Plane perpendicular to $Z$ axis is defined as $X Y$ plane. The length of the pipe routing area along the $\mathrm{Z}$ axis is $\mathrm{L}$ as shown in Figure 1(a). Then the pipe routing area can be divided into three-dimensional mesh points by the following methods:

1) Constructing $m$ equidistant cylinder with $Z$ axis as the center line between $\mathrm{C} 1$ and $\mathrm{C} 2$.

2) Constructing $n$ cross sections go through $Z$ axis and the angle between adjacent sections is equal.

3) Constructing $\mathrm{k}$ equidistant sections which is perpendicular to $\mathrm{Z}$ axis and parallel to end plane to grid laying area.

4) The intersections formed by m equidistant cylinders, $n$ cross sections and $\mathrm{k}$ equidistant sections are the discrete $3 \mathrm{D}$ grid point of the routing area, as shown in Figure 1(b).

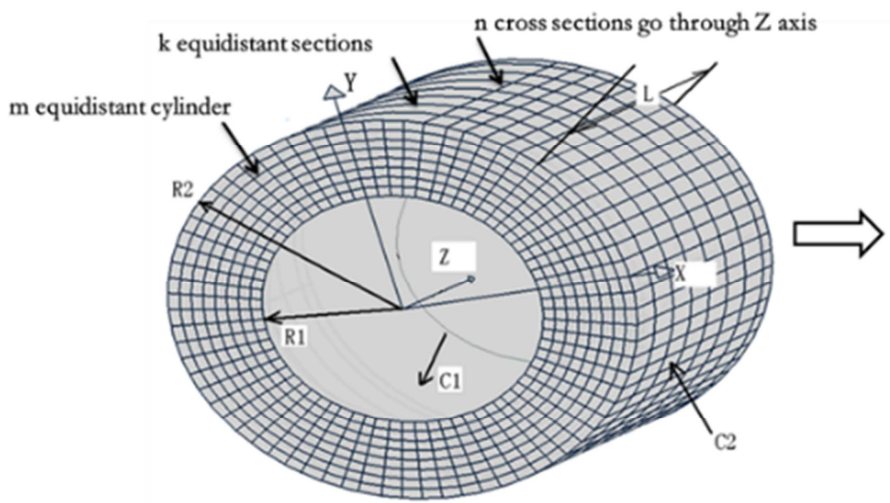

(a)

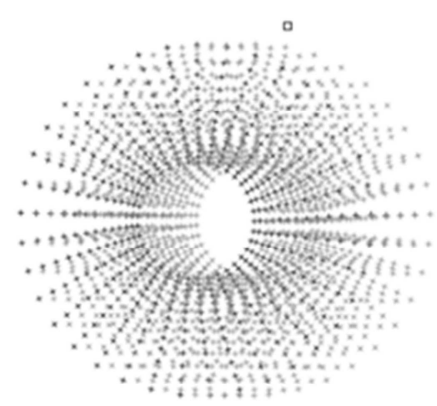

(b)

Figure 1. Gridding of laying area.

Each grid point can be represented by $\left(m^{\prime}, n^{\prime}, k^{\prime}, s\right)$, $m^{\prime}, n^{\prime}$ and $k^{\prime}$ represent the position of the grid point and $\mathrm{s}$ is the status which indicates whether the point is an obstacle. Then the coordinate of the point is as follows:

$$
\left\{\begin{array}{c}
x=\left(R_{1}+\left(R_{2}-R_{1}\right) * \frac{m^{\prime}}{m+1}\right) * \cos \left(2 \pi * n^{\prime} / n\right) \\
y=\left(R_{1}+\left(R_{2}-R_{1}\right) * \frac{m^{\prime}}{m+1}\right) * \sin \left(2 \pi * n^{\prime} / n\right) \\
z=L * k^{\prime} / k
\end{array}\right.
$$

A pipe can be regarded as a polyline composed of several segments from the start point to the end point. The problem discussed in this paper is to find a shortest path for the external pipe routing of aeroengine. The constraints include:

1) The pipe cannot intersect with obstacles.

2) The angle between the two sections of pipeline should not be less than a fixed value considering the machinability of the pipe.

3) Laying the pipe along with axial and circumferential direction as far as possible.

4) The pipe should be straight and short as far as possible.

5) The number of pipe elbows should be as few as possible.

6) The length of a section of the pipe should not be less than 4 times of the pipe diameter.

\section{Algorithm Instruction}

Genetic algorithm is easy to generate illegal solutions for unreasonable coding method, genetic manipulation and a large number of illegal individuals in population. Therefore, this paper uses lee algorithm to generate the initial population and then combines with genetic algorithm to obtain an optimal solution.

\subsection{Lee Algorithm}

Lee algorithm is a traversal algorithm which simulates the propagation process of the wave. This paper defines the start pipe joint as the wave source and the end pipe joint as the target. The diffusion starts from the wave source, and the place where the current wave reaches is called wave front. There is a shortest path from the source to the target when the wave front reaches the target. There will be no path from the wave source to the target if the wave can not reach the target. Lee algorithm has an adaptability, but it needs a large capacity and the 3D space should be changed to $2 \mathrm{D}$ space before pipe routing.

\subsection{Genetic Algorithm}

Genetic algorithm is a stochastic optimization method to simulate the evolution of natural organisms. Each individual represents a potential solution of the problem. Through the manipulation of selecting, crossover and mutation, new individuals are generated and a new population is formed. The optimal individual will be obtained after several iterations.

\section{Solution Strategy}

\subsection{Solving Flow}

Lee algorithm is a traversal algorithm which simulates the 
propagation process of the wave. This paper defines the start pipe joint as the wave source and the end pipe joint as the target. The diffusion starts from the wave source, and the place where the current wave reaches is called wave front. There is a shortest path from the source to the target when the wave front reaches the target. There will be no path from the wave source to the target if the wave can not reach the target. Lee algorithm has an adaptability, but it needs a large capacity and the $3 \mathrm{D}$ space should be changed to $2 \mathrm{D}$ space before pipe routing.

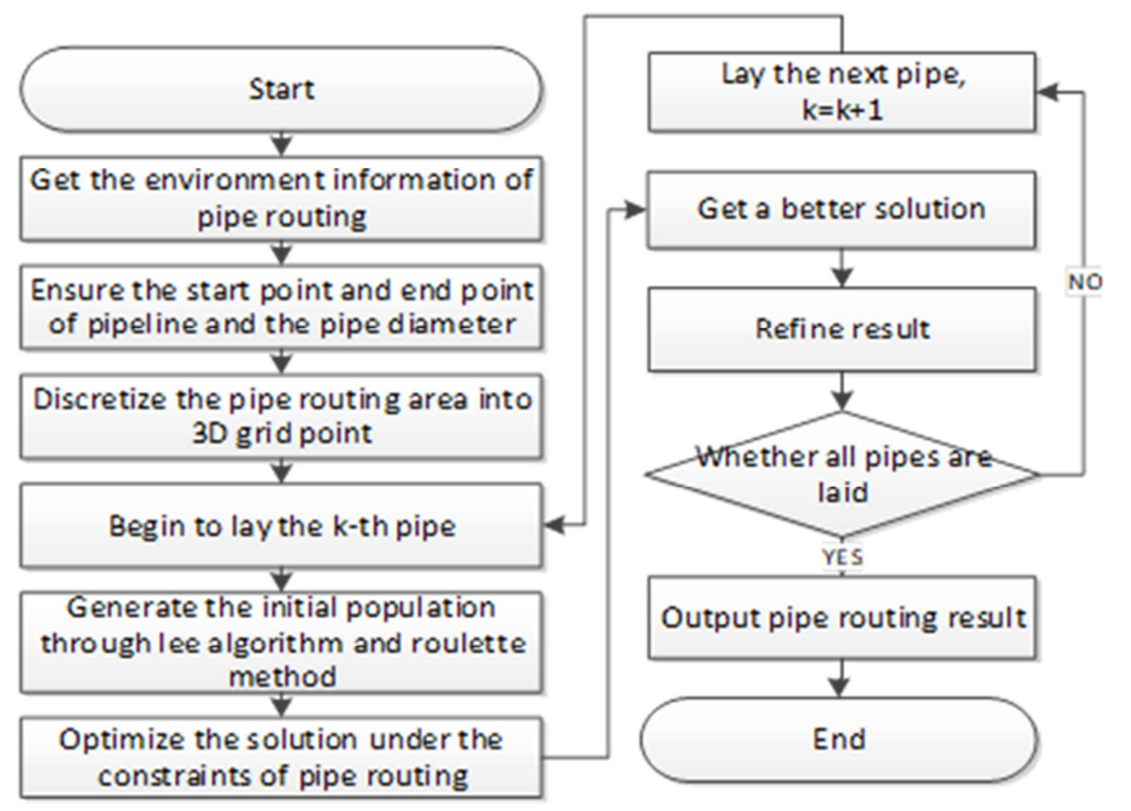

Figure 2. Process of pipe routing.

\subsection{Solving Objective}

The problem discussed in this paper can be expressed as follow:

$$
\max f(k)=\alpha *\left|\frac{l_{\max }-l(k)}{l_{\max }-l_{\min }}\right|+\beta *\left|\frac{w_{\max }-w(k)}{w_{\max }-w_{\min }}\right|+\gamma\left|\frac{o_{\max }-o(k)}{o_{\max }-o_{\min }}\right|
$$

$l(k)$ denotes the length of pipeline when choose the $\mathrm{k}$-th solution, $l_{\max }$ denotes the longest length of pipeline of all solution, $l_{\min }$ denotes the shortest length of pipeline of all solution, $w(k)$ denotes the number of elbows in the pipeline when using the k-th solution, $w_{\max }$ denotes the maximum number of elbows of all solution and $w_{\min }$ denotes the fewest number of elbows of all solution. The direction of the pipeline is divided into axial, circumferential and radial. The number of elbows is measured by the times of pipeline changing its direction. $o(k)$ is a penalty function which denotes whether the constraints are satisfied. $\alpha, \beta, \gamma$ are the weight coefficient of these constraints.

\section{Algorithm Implementation}

\subsection{Chromosome Encoding}

Chromosome encoding is the basis of genetic algorithm which usually uses fixed length binary symbols to represent. A pipeline is composed of several points and segments from the start point to the end point. A large amount of storage space will be wasted when using fixed length binary symbols for the number of points and segments is not fixed. Therefore, the variable length encoding scheme based on chain table is used for chromosome encoding. As shown in Figure 3, each chromosome is represented by a link list and the position of
$3 \mathrm{D}$ grid point and the direction of the next point is stored in the node of the list which can be expressed as $\left\{m_{i}, n_{i}, k_{i}, d i r_{i}\right\}$, $m_{i}, n_{i}$ and $k_{i}$ denote the position and $\operatorname{dir}_{i}$ denotes the direction.

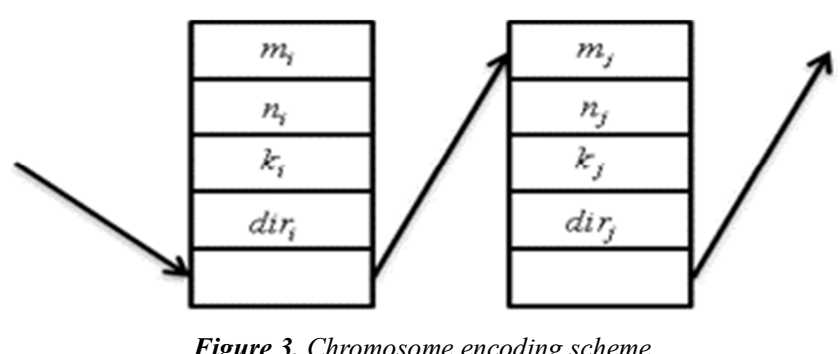

\subsection{Population Initialization}

The productive method on the initial population based on lee algorithm and roulette is proposed in this paper. Lee algorithm determines the process of pipeline generation which bypasses obstacles. Roulette determines the direction of pipeline to ensure the diversity of the population generated by lee algorithm. The initial population generated by this method solves the problem that genetic algorithm is easy to generate illegal individuals to lead repair work. The steps of generating initial population are as follows: 
Step 1. Setting up an array of 3D grid points of pipe environment.

Step 2. Getting the start point sp and the end point tp of the pipeline.

Step 3. Defining a link list. Setting current wave front as $n p$ and previous wave front as $p p$, and putting them into plist $_{i}$ according to lee algorithm.

Step 4. Calculating the probability $p$ of $p p$ moves to $n p$. $p$ is determined by three factors:

1) The relative position of $n p$ with the target point that $p p$ has the tendency to move to the target point; 2) The previous moving direction of $p p$; 3) $p p$ moves to avoid obstacles. The moving direction of $p p$ is shown in Figure 4.

$$
p(n p)=\left(p_{1}(n p)+p_{2}(n p)+p_{3}(n p)\right) * f(n p)
$$

There are six points around $p p$. If there are $m$ of them are obstacles and $n$ of them are closer to $t p$ than $p p$ to $t p$ Then $p_{1}(n p)=0.6 /(6-m)$, which denotes the basic probability of $p p$ moves to $n p$ without considering various constraints. $p_{2}(n p)=0.3 /(3-n)$ if $n p$ is closer to $t p$ than $p p$, else $p_{2}(n p)=0 \cdot p_{3}(n p)=0.1$ if the moving direction of $p p$ to $t p$ is consistent with the previous moving direction, else $p_{3}(n p)=0$. If $n p$ is an obstacle, then $f(n p)=1$, else $f(n p)=0$.

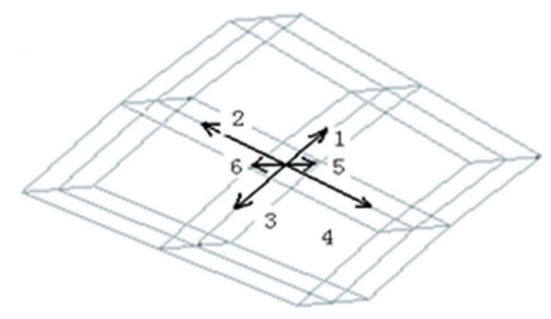

Figure 4. Chromosome encoding scheme.

Step 5. Using Roulette method to determine the moving direction of $p p$ and the next wave front $n p$ with $p(n p)$.

Step 6. An individual of the initial population will be generated if $n p$ is the end point, else go to step 4.

Step 7. Judging whether the number of the population meets the needs of the initial population. If it does, finish the creation of the initial population, else go to step 3.

All individuals of the initial population generated by this method meet the constraints of obstacle avoidance which establishes a good foundation for the genetic manipulation.

\subsection{Fitness Function}

Genetic algorithm uses the individual fitness to decide which individual is inherited to the next population. Fitness function is the key factor for genetic algorithm to get the optimal solution. The fitness function adopted in this paper is as bellow:

$$
F(k)=f(k)
$$

\subsection{Genetic Manipulation}

1) Selection

The probability of individual $k$ being selected is as follows when the population size is $N$ :

$$
p(k)=F(k) / \sum_{j=1}^{N} F_{j}(k)
$$

2) Crossover

For the special way of encoding pipeline and the relationship between the chromosome genes, a special crossover method should be taken which is describe as follows:

Step 1. Choosing two individuals $N 1$ and N2 which need to do crossover operation.

Step 2. Choosing an intersection $G$ from $N 1$.

Step 3. If $G$ exists in $N 2$, exchanging the second half behind $G$ of $N 1$ and $N 2$, as shown in Figure 5, else choosing point $M$ in $N 2$, constructing a path between $G$ and $M$, finally exchanging the second half of $N 1$ and $N 2$ as shown in Figure 6.

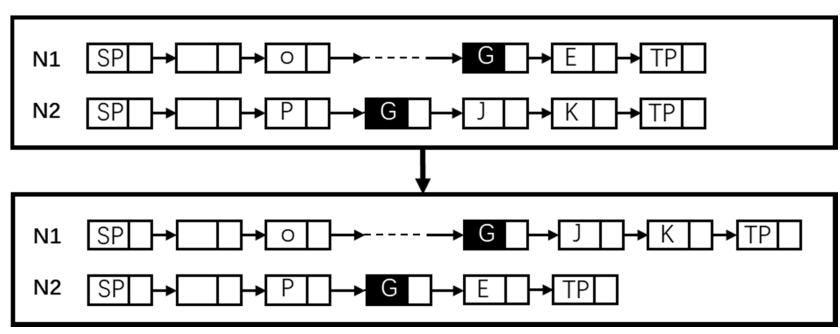

Figure 5. Crossing with same point.

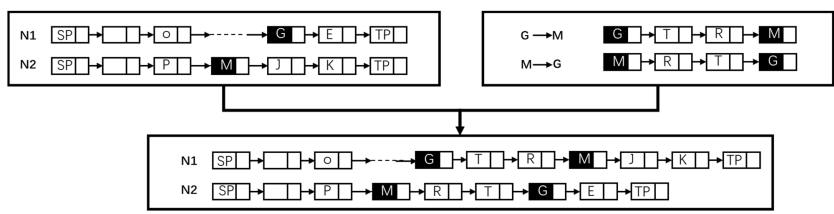

Figure 6. Crossing without same point.

3) mutation

To keep the diversity of the population, the mutation operation is designed in this paper. The mutation manipulation is as follows:

Step 1. Choosing an individual from a population.

Step 2. Choosing two nodes of $O$ and $G$ from the individual as shown in Figure 7.

Step 3. Reconstructing the path between $O$ and $G$ and using it to replace the original path.

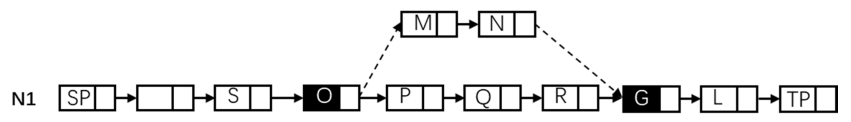

Figure 7. Mutation manipulation.

\section{Case Verification}

Under the platform of $\mathrm{UG}$, an automatic pipe routing module based on lee algorithm and genetic algorithm with $\mathrm{VC}++$ is developed. The module is verified with a simplified model of aircraft engine. Figure 8 shows the result of pipe routing using the automatic pipe laying module. The result shows that the module realized the automatic pipe routing and it mainly solves the problems as follows: 
1) The variable length encoding scheme based on chain table is adopted which is advantageous in expressing the individual information and carrying out the genetic manipulation and mutation manipulation.

2) It solves the problem of the genetic algorithm is easy to generate a large number of illegal solutions when it is used in the area of pipe routing by using the lee algorithm to generate the initial population, which ensures the feasibility of the individuals and optimizes the genetic operation to get the approximately optimal solution more quickly.

3) It solves the problem of population diversity by combined the lee algorithm and roulette method to ensure the quality and diversity of the initial population.

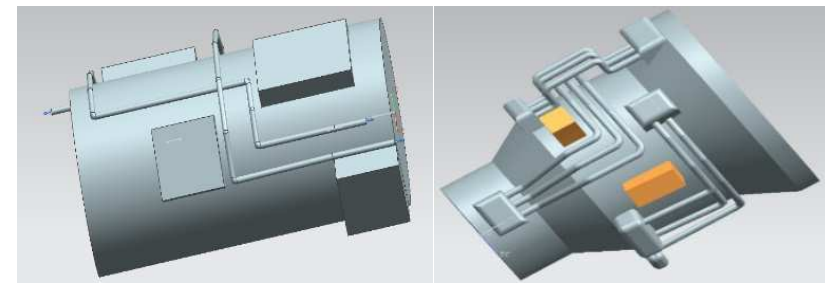

Figure 8. Instance of automatic pipe laying.

\section{References}

[1] CHENG Guang. Structure analysis of Aeroengine Design. Beijing: Beihang University press, 2006.

[2] FAN Jiang, MA Mei, YANG Xiaoguang. Research on automatic laying out for external pipeline of aeroengine $[\mathrm{J}]$. Journal of Machine Design, 2003, 20 (7): 21-23.

[3] LIU Yuanpeng. Research on Key Techniques of Feature-Based Reverse Engineering System for Modeling of Aeroengine Pipelines [D]. Xi'an: Northwestern Polytechnical University, 2006.

[4] Pourke P W. Development of a three-dimensional pipe routing algorithm [D]. Benthlehem: Lehigh University, 1975.

[5] CHEN Zhiying, FAN Jiang, CAI Pangpang, et al. Applied Research on Intelligent Pipe Routing [J]. Mechanical science and technology, 2006, 25 (8): 992-994.

[6] VANDER VELDEN C, BIL C, YU Xinghuo, et al. An intelligent system for automatic layout routing in aerospace design [J]. Innovations in Systems and Software Engineering, 2007, 3 (2): 117-128.

[7] FAN Xiao-ning. A study of optimization methods for ship pipe routing design and applications [D]. Dalian: Dalian University of Technology, 2006.
[8] Belov G, Czauderna T, Dzaferovic A, et al. An Optimization Model for 3D Pipe Routing with Flexibility Constraints [C]. 2017: 321-337.

[9] Lv Yanjie, Zhao Gang. UG-based research and development of 3D pipe layout system of the aircraft engine [J]. Procedia Engineering, 2011: 660-667.

[10] Dong Zongran, Lin Yan. Automatic ship pipe routing method based on shortest pathfaster algorithm [J]. Computer Integrated Manufacturing Systems. 2014, 20 (12): 2962-2972.

[11] Dong Zongran. Research on the method and application of automatic ship pipe routing [D]. 2017.

[12] Yin Y H, Xu L D, Bi Z, et al. A Novel Human-Machine Collaborative Interface for Aero-Engine Pipe Routing [J]. IEEE Transactions on Industrial Informatics, 2013, 9 (4): 2187-2199.

[13] Zhang Y, Bai X L. Research on the Automatic and Optimized Pipe Routing Layout for Aero-Engines Based on Improved Artificial Fish Swarm Algorithm [J]. Applied Mechanics and Materials, 2013, 437: 275-280.

[14] ZHANG Yu, BAI Xiao-lan. Intelligent Multi-pipes Layout for Aero-Engine Based on CAFSC Algorithm [J]. Journal of Northeastern University Natural Science, 2016, 37 (5): 683-687.

[15] Liu Q, Wang C E. A graph-based pipe routing algorithm in aero-engine rotational space $[\mathrm{J}]$. Journal of Intelligent Manufacturing, 2015, 26 (6): 1077-1083.

[16] Zhang Y, Bai X L. The intelligent pipe-routing layout for the aero-engine based on improved artificial fish swarm algorithm [J]. Sensors and Transducers, 2013, 25 (12): 147-154.

[17] Liu Jian-hua, Liu Shao-li, Ning Ru-xin, et al. Integrated technology digital pipeline routing, manufacturing and inspection [J]. Computer Integrated Manufacturing Systems, 2015, 21 (4): 941-954.

[18] QIU Wei. Research on VLSL net routing based on tabu-ant colonies system [D]. Zhenjiang: Jiangsu University, 2009.

[19] LIU Qiang, WANG Cheng-en, REN Tao, et al. POS-based pipe routing approach for aircraft engine [J]. Journal of Noreastern University, 2009, 30 (7): 940-943.

[20] LIU Qiang, WANG Chen-en, BAI Xiaolan. Engineering Rules-based Pipe Routing Algorithm for Aero-engines [J]. Journal of Mechanical Engineering, 2011, 47 (5): 163-168.

[21] Ito T. A genetic algorithm approach to piping route path planning [J]. Journal of Intelligent Manufacturing, 1999, 10: $103-114$.

[22] Lee CY. An algorithm for path connections and its applications. IRE Transactions on Electronic Computers, 1961, EC-10: 346-356. 